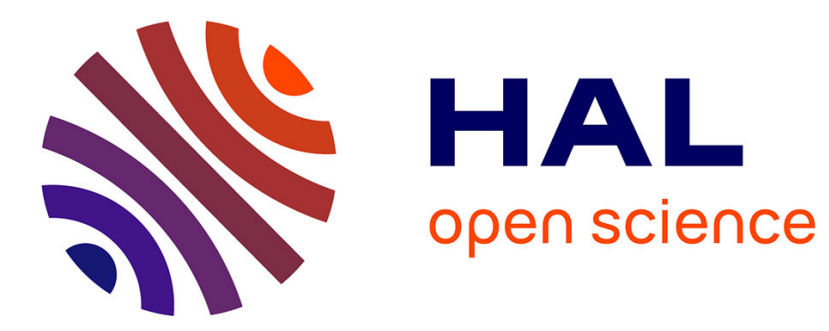

\title{
Identifiability Properties for Inverse Problems in EEG Data Processing and Medical Engineering with Observability and Optimization Issues
}

\author{
Juliette Leblond
}

\section{- To cite this version:}

Juliette Leblond. Identifiability Properties for Inverse Problems in EEG Data Processing and Medical Engineering with Observability and Optimization Issues. Acta Applicandae Mathematicae, 2014, 135 (1), pp. 175-190. 10.1007/s10440-014-9951-7 . hal-00875006v2

\section{HAL Id: hal-00875006 https://hal.science/hal-00875006v2}

Submitted on 5 Jan 2015

HAL is a multi-disciplinary open access archive for the deposit and dissemination of scientific research documents, whether they are published or not. The documents may come from teaching and research institutions in France or abroad, or from public or private research centers.
L'archive ouverte pluridisciplinaire HAL, est destinée au dépôt et à la diffusion de documents scientifiques de niveau recherche, publiés ou non, émanant des établissements d'enseignement et de recherche français ou étrangers, des laboratoires publics ou privés. 


\title{
Identifiability properties for inverse problems in EEG data processing with observability and optimization issues
}

\author{
Juliette Leblond*
}

\begin{abstract}
We consider inverse problems of source identification in electroencephalography, modelled by elliptic partial differential equations. Being given boundary data that consist in values of the current flux and of the electric potential on the scalp, the aim is to reconstruct unknown current sources supported within the brain. For spherical layered models of the head, and after a preliminary data transmission step, such inverse source problems are tackled using best rational approximation techniques on planar sections. Both theoretical and constructive aspects are described, while numerical illustrations are provided.
\end{abstract}

Keywords: Inverse boundary value problems, elliptic partial differential equation, medical imaging, EEG (electroencephalography), observability, optimization.

\section{Introduction}

We discuss some inverse identification problems that arise in medical engineering or in neurosciences for functional and clinical brain analysis purposes. We focus on source recovery issues from boundary data in electroencephalography (EEG). Maxwell's equations are to the effect that the electric potential within the head can be modelled as a solution to some partial differential equation (PDE), in spherical or more general 3-dimensional domains [14]. In particular, with the quasi-static assumption (time derivatives of the electromagnetic fields are neglected), the EEG problem is modelled by an elliptic Poisson-Laplace PDE that only involves the space variable. Boundary data are furnished by a number of pointwise values of the electric potential on the scalp (measured by electrodes on a part of the scalp, see figure 1), together with the (vanishing) current flux. From such partial and overdetermined boundary measurements of the current flux and the potential, the aim is to identify and to reconstruct:

- non-measured boundary data (a Cauchy transmission problem, cortical mapping step),

\footnotetext{
*Team APICS, INRIA Sophia Antipolis, BP 93, 06902 Sophia Antipolis Cedex, France, tel.: +33492387 876, fax: +33492387 858, email: juliette.leblond@inria.fr
} 
- unknown current sources supported within the brain (singularities of the potential), that correspond to the primary cerebral current.

These questions can be rephrased as identification or observation issues for infinite dimensional systems, where the given boundary measurements (flux and potential) coincide with the input and output of the system, of which the electric potential and the current flux inside the head should be viewed as the state. We consider below these inverse potential problems [13]. Related considerations in magnetoencephalography (MEG) will be briefly discussed in conclusion, with others from electric impedance tomography (EIT). Observe further that similar deconvolution issues also appear in automatic control (on the boundary of domains of dimension 2, however), concerning harmonic identification in frequency domain [5].

For dipolar point sources, we review some identifiability results related to the EEG inverse problem [9], that we also formulate as observability properties. Algorithmical and numerical aspects are described, most of them requiring (best constrained quadratic) optimization techniques. Our approach relies on harmonic analysis and function theory (the link with holomorphy comes from harmonicity), as does the work [15]. Compared to other methods (dipole fitting, MUSIC algorithms, [18]), it has the desired feature of providing an estimate of the number of sources (sources that may be correlated, in time).

The overview of the article is as follows. Some notation and definitions are given in section 2. Models and inverse problems in EEG are discussed in section 3. Section 4 is devoted to a two step resolution scheme, which consists first in data transmission (section 4.1), then in source identification (section 4.2). A conclusion is proposed in section 5 .

\section{Notation, definitions}

We recall the definitions of gradient, divergence and Laplace operators for functions acting on $\mathbb{R}^{3}$, where the space variable is denoted by $x=\left(x_{1}, x_{2}, x_{3}\right)$ and the inner product by ".". The gradient and divergence operators are formally defined by:

$$
\operatorname{grad}=\nabla=\left(\frac{\partial}{\partial x_{1}}, \frac{\partial}{\partial x_{2}}, \frac{\partial}{\partial x_{3}}\right)^{t}, \operatorname{div}=\nabla \cdot,
$$

and the Laplace operator by:

$$
\Delta=\nabla \cdot \nabla=\frac{\partial^{2}}{\partial x_{1}{ }^{2}}+\frac{\partial^{2}}{\partial x_{2}{ }^{2}}+\frac{\partial^{2}}{\partial x_{3}{ }^{2}} .
$$

(div acts on $\mathbb{R}^{3}$-valued smooth functions, while grad and $\Delta$ act on $\mathbb{R}$-valued ones).

We set $\Omega \subset \mathbb{R}^{3}$ to be a bounded domain with smooth boundary, and $n$ the unit outer normal vector on $\partial \Omega$. The normal derivative on $\partial \Omega$ is then defined by:

$$
\frac{\partial u}{\partial n}\left(x_{b}\right)=\lim _{x \rightarrow x_{b} \in \partial \Omega} \nabla u(x) \cdot n\left(x_{b}\right) .
$$


Functional Hilbert Lebesgue and Sobolev spaces, $L^{2}$ and $W^{1,2}$, are classically defined on $\Omega$ or $\partial \Omega$, see e.g. [10], as well as $\mathcal{C}(\bar{\Omega})$.

\section{Models, inverse problems in EEG}

\section{Maxwell equations}

Maxwell equations in electrostatics, under quasi-static assumptions, are to the effect that, if $E$ stands for the electric field, and $\boldsymbol{\Psi}$ for the electric potential in the head [14]:

$$
\nabla \times E=0 \Rightarrow E=-\nabla \Psi \text { (Faraday's law). }
$$

The brain is a non magnetic medium, while it is subject to an electric activity represented by the current density $\mathcal{J}$ which satisfies

$$
\mathcal{J}=\sigma E+\mathbf{J}=-\sigma \nabla \mathbf{\Psi}+\mathbf{J}
$$

if $\mathbf{J}$ stands for the primary cerebral current density and $\sigma$ for the electric conductivity of the head $\Omega \subset \mathbb{R}^{3}$. Hence,

$$
\nabla \cdot \mathcal{J}=0 \text { (charge conservation) } \Rightarrow \nabla \cdot(\sigma \nabla \mathbf{\Psi})=\nabla \cdot \mathbf{J} .
$$

Note that $\mathbf{J}$ is supported in the domain $\Omega_{0} \subsetneq \Omega$ corresponding to the brain (there are no current sources outside the brain).

\section{Partial differential equation}

The electric potential $\boldsymbol{\Psi}=\mathbf{\Psi}(x)$ is a real-valued function (or distribution) of the space variable $x \in \mathbb{R}^{3}$ which is solution to the following second order elliptic PDE (to be understood in distribution or variational sense, see section 4):

$$
\begin{gathered}
\operatorname{div}(\sigma \operatorname{grad} \boldsymbol{\Psi})=\operatorname{div} \mathbf{J} \text { or } \nabla \cdot(\sigma \nabla \boldsymbol{\Psi})=\operatorname{div} \mathbf{J} \text { in } \mathbb{R}^{3}, \\
\text { whence } \sum_{i=1}^{3} \frac{\partial}{\partial x_{i}}\left(\sigma \frac{\partial \boldsymbol{\Psi}}{\partial x_{i}}\right)=\sum_{i=1}^{3} \frac{\partial \mathbf{J}}{\partial x_{i}} \text { or } \nabla \sigma \cdot \nabla \boldsymbol{\Psi}+\sigma \Delta \boldsymbol{\Psi}=\nabla \cdot \mathbf{J},
\end{gathered}
$$

for the function or distribution $\mathbf{J}$ with values in $\mathbb{R}^{3}$ and supported in the proper subset $\Omega_{0}$ of $\Omega$ with smooth boundary $\partial \Omega_{0}$ (and such that $\overline{\Omega_{0}} \subset \Omega$ ). Note that the source distribution $\operatorname{div} \mathbf{J}$ is real-valued (or acts on real-valued functions). In EEG, and in the present work as well, $\sigma$ is often assumed to be isotropic (real-valued) and piecewise constant whence the above PDE reduces to a set of Laplace-Poisson equations (see equations (3) below).

\section{Inverse EEG problem}

The inverse EEG problem consists in recovering $\mathbf{J}$ (at least its support in $\Omega_{0}$ ) in some class of source terms, from available boundary values of a solution $\boldsymbol{\Psi}$ 

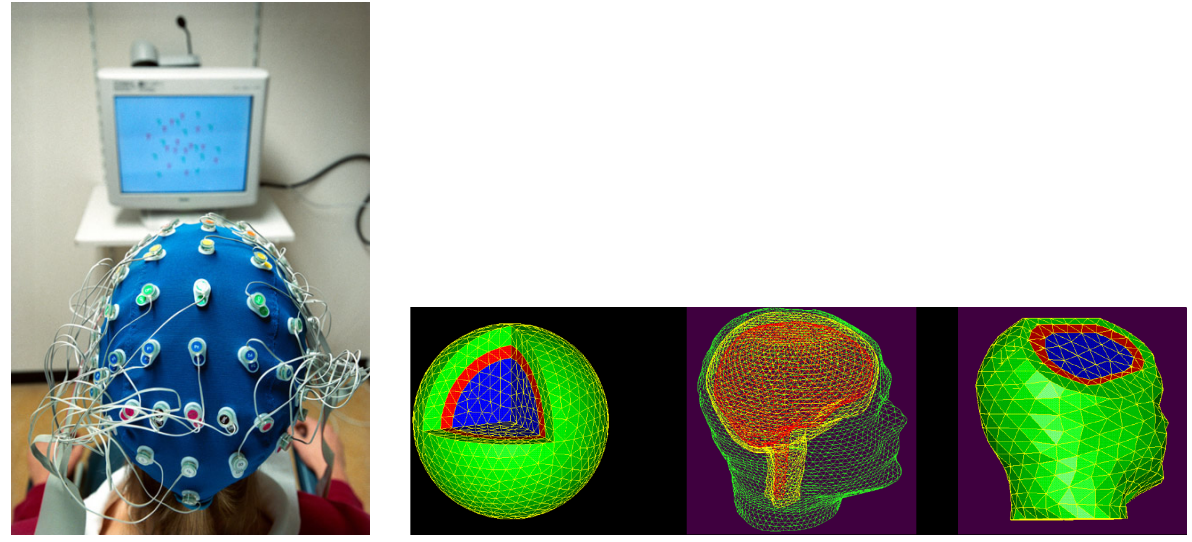

Figure 1: Left (l): measures $\mathbf{u}$ provided by electrodes on the scalp $\partial \Omega$; right $(\mathrm{r})$ : head geometries $\Omega$.

to equation (1):

$$
\mathbf{u}=\frac{\partial \Psi}{\partial n} \text { on } \partial \Omega, \mathbf{y}=\left(\boldsymbol{\Psi}\left(\gamma_{i}\right)\right)^{t}, \gamma_{i} \in \Gamma \subsetneq \partial \Omega, i=1, \cdots, L,
$$

$\mathbf{u}$ being the given current flux on the scalp $\partial \Omega, \mathbf{y}$ the measured potential (or difference of potentials), by $L$ electrodes on the upper part of the scalp, located at positions $\gamma_{i}$ on a part $\Gamma \subsetneq \partial \Omega$ of the boundary (see figure 1, (l)).

The above inverse problem is basically ill-posed, and requires additionnal assumptions concerning $\boldsymbol{\Psi}$ and $\mathbf{J}$ in order to admit a unique solution. Still, stability properties of the solution are difficult to ensure, and only hold under further a priori assumptions on the model and the data [13]. These well-posedness aspects will be discussed within the harmonic framework of section 4 , having in mind that the available measurements $\mathbf{u}, \mathbf{y}$ are incomplete and may be corrupted, in practice.

\section{Direct EEG problem}

Concerning the associated direct problems, the source distribution $\mathbf{J}$ is given (supported in $\Omega_{0}$ ), as well as boundary data of Dirichlet or Neumann type [10]. Dirichlet boundary data consists in the potential $\boldsymbol{\Psi}$ on the overall $\partial \Omega$, while Neumann data are furnished by $\frac{\partial \Psi}{\partial n}$. For smooth conductivities, these problems are well-posed, under the following necessary and sufficient compatibility condition for the second one, with which the solution is unique up to an additive constant:

$$
\iint_{\partial \Omega} \sigma \frac{\partial \Psi}{\partial n} d s=0
$$

with respect to the Lebesgue measure $d s$ on the surface $\partial \Omega$. This is a consequence of Green's formula together with the fact that $\mathbf{J}$ vanishes outside $\Omega_{0}$ 
hence on $\partial \Omega$. In particular, whenever $\boldsymbol{\Psi}$ is smooth enough on $\partial \Omega$, then so is $\boldsymbol{\Psi}$ in $\Omega$. Actually, for smooth or piecewise constant conductivities $\sigma$, the boundary assumption $\boldsymbol{\Psi} \in W^{1,2}(\partial \Omega)$ is enough to ensure that $\boldsymbol{\Psi} \in \mathcal{C}(\bar{\Omega})$, see e.g. [8] for constant $\sigma$.

\section{Observability issues}

The electric potential $\boldsymbol{\Psi}=\boldsymbol{\Psi}(x)$, a real valued function (or distribution) of the space variable $x \in \Omega \subset \mathbb{R}^{3}$ may be viewed as a state variable for the static infinite dimensional state model (1). On the boundary $\partial \Omega$, the current flux $\mathbf{u}$ corresponds to the associated input, the potential $\mathbf{y}$ to the output. The inverse source problem consists in finding the state or its singularities, given input/output data $\mathbf{u}$ and $\mathbf{y}$, which is an observability problem (in general, for EEG, $\mathbf{u}$ is assumed to vanish).

At this stage, we directly get from (2) that:

$$
\mathbf{y}=C\left(\boldsymbol{\Psi}_{\mid \partial \Omega}\right),
$$

where $C$ denotes the pointwise evaluation operator at the $L$ points $\gamma_{i} \in \Gamma \subsetneq$ $\partial \Omega$, and corresponds to an observation operator. With the above smoothness assumptions, the linear operator $C$ is continuous, and it holds that:

$$
|\mathbf{y}| \lesssim\|\Psi\|_{L^{\infty}(\partial \Omega)} \lesssim\|\mathbf{u}\|_{L^{\infty}(\partial \Omega)} .
$$

Note that $C$ has finite dimensional range and is formally defined on those continuous functions on $\partial \Omega$. Without further assumption, the reconstruction of the infinite dimensional state $\boldsymbol{\Psi}$ within the head with very few observations (the boundary measurements $\mathbf{y}$ ) is lost in advance. We will see that it goes differently under suitable hypotheses, and that some quantities become observable, at least approximately.

Similarly, the relations (1) and (2) may be expressed through Green formula, see (5) and (8), as:

$$
\mathbf{u}=K_{\mathbf{J}}\left(\boldsymbol{\Psi}_{\mid \partial \Omega}\right), \text { where } K_{\mathbf{J}}: \boldsymbol{\Psi}_{\mid \partial \Omega} \mapsto{\frac{\partial \boldsymbol{\Psi}}{\partial n}{ }_{\mid \partial \Omega}},
$$

for the so-called Dirichlet-to-Neumann operator $K_{\mathbf{J}}$. In the present situation, a preliminary step is required in order to build $\boldsymbol{\Psi}$ on $\partial \Omega$ from $\mathbf{y}$, a step which would not be needed if the measurements $\mathbf{y}$ were available on the whole boundary $\partial \Omega$, rather than at points in $\Gamma \subsetneq \partial \Omega$. Nevertheless, this only reinforces the strong ill-posedness property of the corresponding observability issue, of building the state $\boldsymbol{\Psi}$ on $\Omega$ from $\mathbf{u}$ and $\mathbf{y}$ on $\partial \Omega$, but unknown $\mathbf{J}$, among solutions to (1), an impossible task. Regularization schemes by constrained optimization (best quadratic approximation) are then used in order to state and to solve these inversion issues in several consecutive steps. 

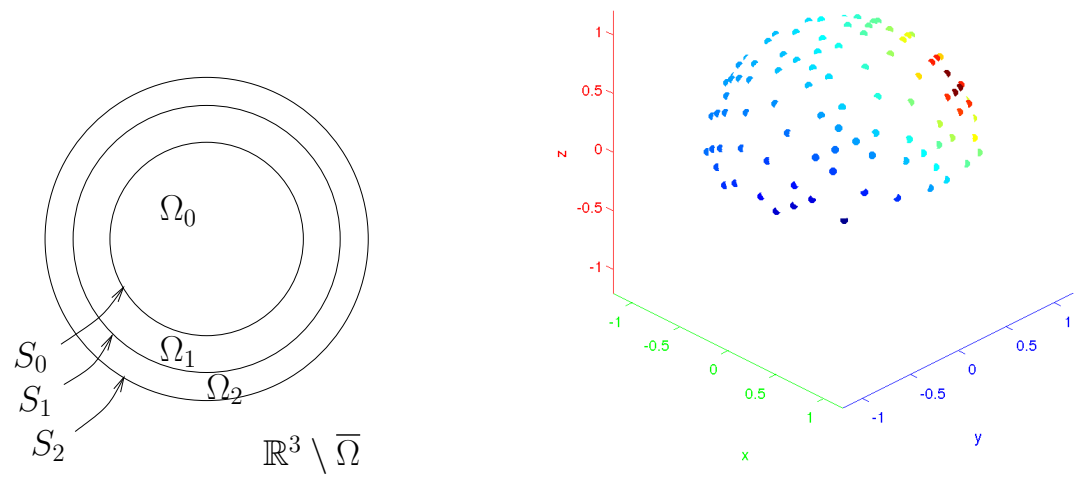

Figure 2: (l): 3 layers spherical head model (planar cross section); (r) : measured values $\mathbf{y}=\mathbf{y}_{2}$ of $\boldsymbol{\Psi}$ by $L=128$ electrodes on the upper part of the scalp, $\left(x_{1}, x_{2}, x_{3}\right)$ coordinates.

\section{EEG inverse source problem}

Spherical head models are classically considered and supposed to be made of 3 spherical homogeneous layers [9]. Put then $\Omega=\mathbb{B}$ for the unit ball and $\partial \Omega=\mathbb{S}$ for the unit sphere. Put $\Omega_{0}=r_{0} \mathbb{B}$ for some $0<r_{0}<1$ (brain), $\Omega_{1}$ (skull), $\Omega_{2}$ (scalp), such that $\Omega=\Omega_{0} \cup \overline{\Omega_{1}} \cup \Omega_{2}$, with $\partial \Omega_{i}=S_{i-1} \cup S_{i}$ for $i=1,2$ and spheres $S_{i}$, see figure 2, (l). In particular, we get $S_{0}=\partial \Omega_{0}=r_{0} \mathbb{S}$ and $S_{2}=\partial \Omega=\mathbb{S}$.

The head conductivity $\sigma$ is assumed to be known and piecewise constant: on $\Omega_{k}, \sigma=\sigma_{k}>0$ (with $\sigma_{0}=\sigma_{2}=1$ up to a renormalization, and $1 / \sigma_{1} \in[20,80]$ ). Further, because $\mathbb{R}^{3} \backslash \bar{\Omega}$ (the air, the neck is ignored) is a non conductive medium, we have that $\sigma$ vanishes outside $\Omega$.

Given $\mathbf{u}$ and $\mathbf{y}$ on $\partial \Omega$ from (2) (see figure 2, (r)), we thus want to find $\mathbf{J}$ or at least its support, such that $\boldsymbol{\Psi}$ satisfies (1). Of course, necessary assumptions are needed to ensure well-posedness and observability properties, like hypothesis (9) below, to the effect that $\mathbf{J}$ is a finite sum of pointwise dipolar sources. In particular, we want to locate the singularities of $\boldsymbol{\Psi}$ in $\Omega_{0}$. More precisely, we get from (1) that:

$$
\left\{\begin{array}{l}
\Delta \boldsymbol{\Psi}=0 \text { in }\left(\mathbb{R}^{3} \backslash \bar{\Omega}\right) \cup \Omega_{2} \cup \Omega_{1}, \\
\Delta \boldsymbol{\Psi}=\operatorname{div} \mathbf{J} \text { in } \Omega_{0}, \\
\boldsymbol{\Psi} \text { and } \sigma \frac{\partial \boldsymbol{\Psi}}{\partial n} \text { continuous across } S_{i}, i=0,1,2 .
\end{array}\right.
$$

The above transmission conditions (obtained from Green formula, see (5) below) 
express the continuity of the potential and of the normal current across the interfaces $S_{i}$. We use two main consecutive steps for solving the EEG inverse source problem [9]:

- A first boundary data extension / transmission step (Cauchy type inverse problem), also called "cortical mapping" step, in the present framework: the given boundary data are transmitted from $\partial \Omega=S_{2}$ (scalp) to $S_{0}$ (cortex), see section 4.1 .

- A second source localization step, in $\Omega_{0}$, for some class of $\mathbf{J}$ (geometric inverse problem): from the above transmitted data on $S_{0}$, locate the sources inside $\Omega_{0}$, see section 4.2 .

\subsection{Data transmission}

Let $S_{i}^{ \pm}$denote the inner and outer sides of $S_{i}$, for $i=0,1,2$. From (3), we get in the outermost two layers $\Omega_{i}, i=1,2$, with the convention $\sigma_{3}=0$ :

$$
\left\{\begin{array}{l}
\Delta \Psi=0 \text { in } \Omega_{i}, i=1,2, \\
\boldsymbol{\Psi}_{\left.\right|_{S_{i}^{-}}}=\boldsymbol{\Psi}_{\left.\right|_{S_{i}^{+}}},\left.\sigma_{i} \frac{\partial \boldsymbol{\Psi}}{\partial n}\right|_{S_{i}^{-}}=\left.\sigma_{i+1} \frac{\partial \boldsymbol{\Psi}}{\partial n}\right|_{S_{i}^{+}} .
\end{array}\right.
$$

In order to get the Cauchy data on $S_{0}$, we thus face two consecutive Cauchy type transmission problems in the spherical shells $\Omega_{i}$, from their outer boundaries $S_{i}^{-}$to their inner ones $S_{i-1}^{+}$. Put $\mathbf{y}=\mathbf{y}_{2}, \mathbf{u}=\mathbf{u}_{2}=0$. The first transmission problem is the following. Given $\mathbf{y}_{2} \in \mathbb{R}^{L}$ such that:

$$
\left\{\begin{array}{l}
\Delta \boldsymbol{\Psi}=0 \text { in } \Omega_{2} \\
\left(\boldsymbol{\Psi}\left(\gamma_{i}\right)\right)^{t}=\mathbf{y}_{2} \in \mathbb{R}^{L}, \gamma_{i} \in \Gamma \subset S_{2}, i=1, \cdots, L \\
\left.\frac{\partial \boldsymbol{\Psi}}{\partial n}\right|_{S_{2}^{-}}=\mathbf{u}_{2}=0
\end{array}\right.
$$

get on $S_{1}^{+}$:

$$
\mathbf{y}_{1}=\Psi_{\left.\right|_{S_{1}^{+}}} \text {and } \mathbf{u}_{1}=\left.\frac{\partial \boldsymbol{\Psi}}{\partial n}\right|_{S_{1}^{+}},
$$

recalling the normalization $\sigma_{2}=1$. Once $\mathbf{u}_{1}$ and $\mathbf{y}_{1}$ have been computed on $S_{1}^{+}$(either by their pointwise values at points from a mesh or by their spherical harmonics expansions [10]), the second transmission problem in $\Omega_{1}$ can be stated as follows. Given $\mathbf{u}_{1}, \mathbf{y}_{1}$ on $S_{1}^{-}$such that:

$$
\Delta \boldsymbol{\Psi}=0 \text { in } \Omega_{1}, \boldsymbol{\Psi}_{\left.\right|_{S_{1}^{-}}}=\mathbf{y}_{1},\left.\sigma_{1} \frac{\partial \boldsymbol{\Psi}}{\partial n}\right|_{S_{1}^{-}}=\mathbf{u}_{1}
$$

get on $S_{0}$ :

$$
\mathbf{y}_{0}=\Psi_{\left.\right|_{S_{0}^{+}}} \text {and } \mathbf{u}_{0}=\left.\sigma_{1} \frac{\partial \boldsymbol{\Psi}}{\partial n}\right|_{S_{0}^{+}}
$$


Cauchy-Holmgren uniqueness result asserts that, for compatible (exact) data, there exists a unique solution to the above transmission problem. Ill-posedness, however, comes from unstability properties of such Cauchy type issues, though sufficient conditions for stability are available [1], [21]. As soon as we turn to experimental (corrupted) data, an exact solution may not even exist.

However, robust approximated identifiability / observability properties can be ensured as follows, by regularization and approximation techniques, from which constructive resolution schemes are derived. Let $E_{3}$ be the radial fundamental solution of Laplace equation in $\mathbb{R}^{3}$, see [10]:

$$
E_{3}(x)=-\frac{1}{4 \pi|x|}, \text { which satisfies } \Delta E_{3}=\delta_{0} \text { on } \mathbb{R}^{3},
$$

if $\delta_{C}$ stands for the Dirac distribution (mass) at point $C$. Using Green formula for harmonic functions, we get that for $x \notin \Omega_{i}$ and $i=1,2$ :

$$
\iint_{\partial \Omega_{i}}\left(\boldsymbol{\Psi}(y) \frac{\partial E_{3}}{\partial n}(x-y)-E_{3}(x-y) \frac{\partial \Psi}{\partial n}(y)\right) d s(y)=0,
$$

where

$$
\frac{\partial E_{3}}{\partial n}(x-y)=\frac{(x-y) \cdot n(x)}{4 \pi|x-y|^{3}} .
$$

To handle this cortical mapping step, we use boundary elements methods (BEM) described in [9]. The quantities $\boldsymbol{\Psi}, \frac{\partial \boldsymbol{\Psi}}{\partial n}$ are discretized on the meshes and represented as a (big) vector $\Psi$ which represents $\left(\mathbf{u}_{i}, \mathbf{y}_{i}\right)$ at points on the spheres $S_{i}, i=0,1,2$. We then look for a vector $\Psi$ such that $M \Psi=\left(\mathbf{u}_{2}, \mathbf{y}_{2}\right)$ (the given data), for a measurement matrix $M$ (depending on the meshes). Further, we require that $\Psi$ belongs to the kernel of some matrix $H$, a relation which expresses formula (5) and that links $\mathbf{u}_{i}, \mathbf{y}_{i}$ on $S_{i}$ to $\mathbf{u}_{i-1}, \mathbf{y}_{i-1}$ on $S_{i-1}$, for $i=1,2$. Formula (5) however is solvable only for compatible (exact) data, whence we turn to optimization. This raises the issue of minimizing the following discrete criterion on $\partial \Omega_{i}[16]$ :

$$
\min _{H \Psi=0}\left\|M \Psi-\left(\mathbf{u}_{2}, \mathbf{y}_{2}\right)\right\|_{l^{2}}^{2}+\lambda\|R \Psi\|_{l^{2}}^{2},
$$

for some Lagrange parameter $\lambda>0$ and an appropriate matrix $R$ which expresses the constraints (Tikhonov regularization). This furnishes a regularized resolution scheme, even for non-compatible data. Numerical illustrations are furnished in figure 3 , which represents the transmitted Cauchy data $\mathbf{y}_{0}, \mathbf{u}_{0}$ on the cortex $S_{0}=\partial \Omega_{0}$, at 642 points on the meshed spheres, computed using boundary elements (BEM) from the electrodes pointwise data $\mathbf{y}=\mathbf{y}_{2}$ on the scalp $\partial \Omega_{2}$, see figure 2 . There, and in figures $5,6,7$ as well, we simulated direct data with $\mathbf{J}$ as in (9) and $K=2$ sources $C_{1}=(.5, .5, .5), C_{2}=(.5,-.5,-.4)$. All the numerical experiments were obtained using the software FindSources3D (matlab) [12]. Note that related bounded extremal problems (BEP) express a criterion similar to (6), though expressed in $L^{2}\left(\partial \Omega_{i}\right)$ norm, within Hardy classes of gradients of harmonic functions [3]. There, expansions on bases of spherical 

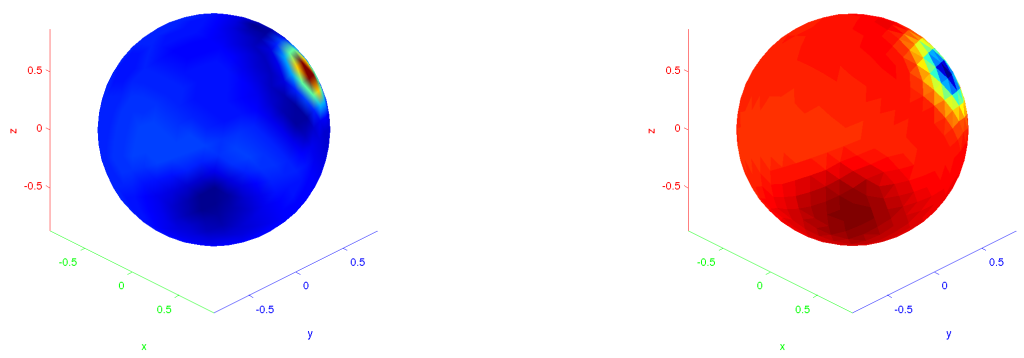

Figure 3: Potential and normal current transmitted on $S_{0},\left(x_{1}, x_{2}, x_{3}\right)$ coordinates: (l) $\mathbf{y}_{0} ;(\mathrm{r}) \mathbf{u}_{0}$.

harmonics may be used rather than pointwise values for the discretization. In both cases, robust solutions are furnished from best quadratic approximation of the given boundary data, using discretizations of the Laplace operator (BEM) or expansions of harmonic functions (BEP), together with regularizing norm constraints.

\subsection{Source identification}

From the cortical data $\mathbf{y}_{0}, \mathbf{u}_{0}$ on $S_{0}$, the inverse source problem consists in finding the distribution $\mathbf{J}$ (or its support inside the ball $\Omega_{0}$ ) such that:

$$
\left\{\begin{array}{l}
\Delta \boldsymbol{\Psi}=\operatorname{div} \mathbf{J} \text { in } \Omega_{0}, \\
\boldsymbol{\Psi}_{\left.\right|_{S_{0}}}=\mathbf{y}_{0},\left.\quad \frac{\partial \boldsymbol{\Psi}}{\partial n}\right|_{S_{0}}=\mathbf{u}_{0} .
\end{array}\right.
$$

Without further assumptions, this is still an ill-posed problem which admits infinitely many solutions $\mathbf{J}$.

The potential $\boldsymbol{\Psi}$ may be expressed in terms of $\mathbf{J}$, by convolution with a fundamental solution of Laplace equation in $\mathbb{R}^{3}$, see [10]. Indeed, we get for $x \in \Omega_{0} \backslash \operatorname{supp} \mathbf{J}:$

$$
\begin{gathered}
\mathbf{\Psi}(x)=h(x)+\iiint_{\Omega_{0}} E_{3}(x-y) \operatorname{div} \mathbf{J}(y) d y \\
=h(x)-\iiint_{\Omega_{0}} \nabla E_{3}(x-y) \cdot \mathbf{J}(y) d y=h(x)+\mathbf{\Psi}_{s}(x),
\end{gathered}
$$

for some function $h$ harmonic in $\Omega_{0}$, where $\boldsymbol{\Psi}_{s}$ represents the singular part of $\boldsymbol{\Psi}$ and contains all information about the source term. Note that $\boldsymbol{\Psi}_{s}$ is harmonic outside $\Omega_{0}$ and vanishes at $\infty$; it can be computed on $S_{0}$ from $\mathbf{u}_{0}$ and $\mathbf{y}_{0}$ expanded on the spherical harmonic basis [10]. 


\subsubsection{Dipolar point sources}

The following hypothesis on $\mathbf{J}$ is classical in EEG, which amounts to assume that the potential $\boldsymbol{\Psi}$ is created by $K$ dipolar point sources $C_{k} \in \Omega_{0}$ with associated moments $p_{k} \in \mathbb{R}^{3}$ :

$$
\mathbf{J}=\sum_{k=1}^{K} p_{k} \delta_{C_{k}}, \text { whence } \Delta \boldsymbol{\Psi}=\operatorname{div} \mathbf{J}=\sum_{k=1}^{K} p_{k} \cdot \nabla \delta_{C_{k}}
$$

In this situation, we get from (4), (8), at $x \neq C_{k}$ in $\mathbb{R}^{3}$ :

$$
\boldsymbol{\Psi}_{s}(x)=\frac{-1}{4 \pi} \sum_{k=1}^{K} \frac{p_{k} \cdot\left(x-C_{k}\right)}{\left|x-C_{k}\right|^{3}} .
$$

It ensures well-posedness of the above homogeneous inverse source problem (7), with unknown $K, C_{k}, p_{k}$, from Dirichlet-Neumann data on $S_{0}$ [20], [21]. As a consequence, source identifiability properties from scalp boundary data on $\partial \Omega$ hold true for the inverse EEG problem (1), (2), provided that the Dirichlet data $\mathbf{y}_{2}$ is furnished on an open subset $\Gamma \subset \partial \Omega$. Whenever the potential values are only given at $L$ points $\gamma_{i} \in \Gamma$, which is practically the case, a first robust interpolation step is thus required. Uniqueness of $\mathbf{J}$ in the above class, hence of $K, p_{k}, C_{k}$, for $k=1, \cdots, K$, is established in [11]. Such identifiability results from boundary data can be viewed as observability properties. Again, constructive aspects and robust resolution algorithms constitute the key points.

\subsubsection{Source localization scheme}

Given the function $\Psi_{s}$ on $S_{0}$, we now show how to identify the $6 K+1$ real valued quantities that characterize the sources ( $K$ itself, and $C_{k}, p_{k}$, for $k=1, \cdots, K$ ). We assume $\boldsymbol{\Psi}_{s}$ to be either expanded as a series on $S_{0}$ or given by pointwise values at the mesh points there, for computational purposes. The localization algorithm is described in [4], [9]. It consists in singularities estimation by best quadratic rational approximation of $\boldsymbol{\Psi}_{s}$ (actually, of $\boldsymbol{\Psi}_{s}^{2}$ ) on the boundaries (circles) of families of planar sections of $\Omega_{0}$ (disks).

\section{Singularities in planar sections}

Let, for instance, $\Pi=\left\{\left(x_{1}, x_{2}, x_{3}\right), x_{3}=0\right\}$ denote the $\left(x_{1}, x_{2}\right)$ plane, and $\Pi_{p}=\left\{\left(x_{1}, x_{2}, x_{3}\right), x_{3}=x_{3 p}\right\}$, with the disk $D_{p}=\Pi_{p} \cap \Omega_{0}$ and the circle $T_{p}=\partial D_{p}=\Pi_{p} \cap S_{0}, p=1, \cdots, P$, for some integer $P>0$ (see figure 4 ).

From $\boldsymbol{\Psi}_{s}$ on $S_{0}$, for each $p=1, \cdots, P$, build the complex variable functions $f_{p}$ such that, for $z=x_{1}+i x_{2} \in T_{p}$ :

$$
f_{p}(z)=\Psi_{s}^{2}\left(x_{1}, x_{2}, x_{3 p}\right)
$$

It then holds that:

$$
f_{p}(z)=\left[\sum_{k=1}^{K} \frac{\phi_{k p}(z)}{\left(z-z_{k p}\right)^{3 / 2}}\right]^{2}
$$




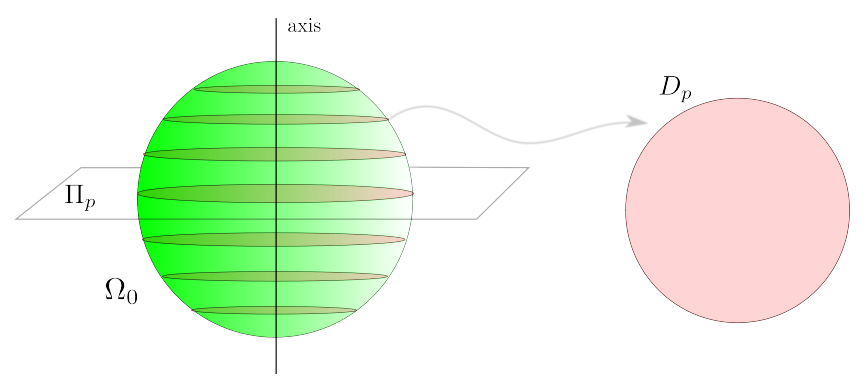

Figure 4: Planar sections $\Pi_{p}=\left\{\left(x_{1}, x_{2}, x_{3 p}\right)\right\}$ of $\Omega_{0} \leadsto$ disks $D_{p} \subset \Pi_{p}, p=$ $1, \cdots, P$.

$$
=\sum_{k=1}^{K} \frac{\phi_{k p}^{2}(z)}{\left(z-z_{k p}\right)^{3}}+\sum_{\substack{j, k=1 \\ j \neq k}}^{K} \frac{2 \phi_{k p}(z) \phi_{j p}(z)}{\left(z-z_{k p}\right)^{3 / 2}\left(z-z_{j p}\right)^{3 / 2}},
$$

for functions $\phi_{k p}$ such that the products $\phi_{k p} \phi_{j p}$ are holomorphic in $D_{p}$ for $j, k=1, \cdots, K$ and complex valued singularities $z_{k p} \in D_{p}$.

Indeed, the denominator of $\boldsymbol{\Psi}_{s}$ involves the quantities $\left|x-C_{k}\right|^{3}$ that can be computed as follows. If we write $C_{k}=\left(x_{1 k}, x_{2 k}, x_{3 k}\right), z_{k}=x_{1 k}+i x_{2 k}$ for the complex affix of $C_{k}$ in $\Pi \cap S_{0} \simeq \mathbb{C}, r_{p}=\sqrt{1-x_{3 k}^{2}}$ and $h_{k p}=x_{3 k}-x_{3 p}$, we get:

$$
\left|x-C_{k}\right|^{2}=\left|z-z_{k}\right|^{2}+h_{k p}^{2}=\left(z-z_{k}\right)\left(\bar{z}-\overline{z_{k}}\right)+h_{k p}^{2} .
$$

When $x \in T_{p}$, then $z \in T_{p}$ and $\bar{z}=r_{p}^{2} / z$, whence

$$
\left|x-C_{k}\right|^{2}=\left(z-z_{k}\right)\left(\frac{r_{p}^{2}}{z}-\overline{z_{k}}\right)+h_{k p}^{2} .
$$

Assume that $z_{k} \neq 0$, which generically holds if $C_{k} \neq 0$ since $z_{k}$ only depends on $C_{k}$ and $\Pi$. Expanding the above rational function of $z$, we find:

$$
-\frac{\overline{z_{k}}}{z}\left(z^{2}-\frac{r_{p}^{2}+h_{k p}^{2}+\left|z_{k}\right|^{2}}{\overline{z_{k}}} z+\frac{z_{k}}{\overline{z_{k}}} r_{p}^{2}\right)=-\frac{\overline{z_{k}}}{z}\left(z-z_{k p}\right)\left(z-z_{k p}^{(r)}\right),
$$

for $z_{k p} \in D_{p}$ and $z_{k p}^{(r)} \notin D_{p}$ such that

$$
\left|z_{k p} z_{k p}^{(r)}\right|=r_{p}^{2},
$$

in particular. This implies that, on $T_{p}$ :

$$
\frac{p_{k} \cdot\left(x-C_{k}\right)}{\left|x-C_{k}\right|^{3}}=\frac{\phi_{k p}(z)}{\left(z-z_{k p}\right)^{3 / 2}}, \text { where } \phi_{k p}(z)=\frac{\sqrt{z} \pi_{k p}(z)}{\left(z-z_{k p}^{(r)}\right)^{3 / 2}},
$$


where $\pi_{k p}$ is a polynomial of degree 2 , which depends on $p_{k}, z_{k}, h_{k p}, r_{p}$. Because $z_{k p}^{(r)} \notin D_{p}$, this shows that $\phi_{k p} \phi_{j p}$ are holomorphic functions in $D_{p}$, for $j, k=$ $1, \cdots, K$, and establishes (10). Observe that the functions $\phi_{k p}$ are multiply valued in $D_{p}$, due to the presence of $\sqrt{z}$ in their numerators, while this is no longer the case for the products $\phi_{k p} \phi_{j p}$; this is the reason why we consider the squared values $\boldsymbol{\Psi}_{s}^{2}$ of $\boldsymbol{\Psi}_{s}$.

As a consequence, we get that for each $p, f_{p}$ coincides on $T_{p}$ with a function that admits $K$ singularities $z_{k p}$ in $D_{p}$. These singularities $z_{k p}$ are due to the sources, and related to their parameters $C_{k}, p_{k}$ (and to $x_{3 p}$ as well). Indeed, assuming that $z_{k} \neq 0$, the following behaviour of $z_{k p}$ can be checked from (11). (i) The complex arguments of the $K$ singularities $\left(z_{k p}\right)$ of $f_{p}$ do not depend on $p$ and coincide with the argument of $z_{k}$ (because $z_{k p} / z_{k}$ are real valued).

(ii) For fixed $k$, the modulus $\left|z_{k p}\right|$ is maximum w.r.t. $p$ in the section $D_{p *}$ closest to (or containing) $C_{k}$, where $z_{k p *}=z_{k}$.
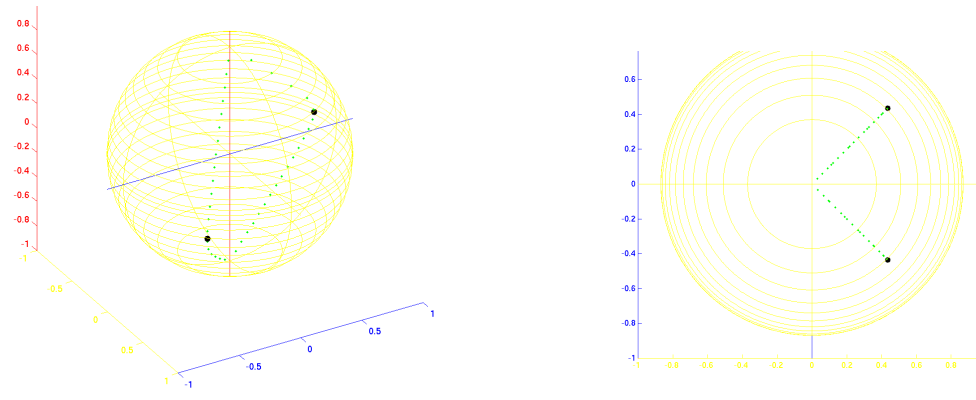

Figure 5: Sources $C_{k}$ in $\Omega_{0}$ (big black dots), singularities $\left(z_{k p}\right)$ (small green dots), for $k=1,2$; left (l): side view, $\left(x_{1}, x_{2}, x_{3}\right)$ coordinates; right $(\mathrm{r})$ : from above (top of $x_{3}$ axis), superimposed $\left(x_{1}, x_{2}, x_{3 p}\right)$ planes .

Whenever $z_{k}=0$, the corresponding term within $f_{p}$ in (11) degenerates and admits a simple pole at $z_{k p}=0$, for $p=1, \cdots, P$. Actually, it behaves as $\pi_{k p} / z$.

These properties are illustrated in figure 5 with $K=2$ sources $C_{1}, C_{2}$ as in section 4.1 and figure 3 , and for $P=21$ sections (where yellow circles correspond to boundary circles $T_{p}$, as in the next figures). They allow us to reduce the $3 \mathrm{D}$ inverse source problem to a family of $2 \mathrm{D}$ boundary value problems, for $p=1, \cdots, P$ : being given $f_{p}$ on the boundary $T_{p}$, recover its $K$ singularities $z_{k p} \in D_{p}$.

\section{Poles of rational approximants}

For fixed $p$, we see from (10) that the singularities $z_{k p} \in D_{p}$ appear both as $K$ triple poles and as $K$ branchpoints of $f_{p}$. It then turns out that they may be 
approximated by the poles in $D_{p}$ of best quadratic rational approximants to $f_{p}$ on $T_{p}$, defined as follows. Consider the best rational approximation (constrained optimization) problem:

For $n \geq 0$, find polynomials $p_{n}, q_{n}$ with degree $p_{n} \leq$ degree $q_{n} \leq n$ and $q_{n}$ with less than $n$ zeroes in $D_{p}$, that minimize

$$
\left\|f_{p}-\frac{p_{n}}{q_{n}}\right\|_{L^{2}\left(T_{p}\right)}
$$

among such functions [6].

Solutions $p_{n} / q_{n}$ are the best quadratic rational approximants to $f_{p}$ on $T_{p}$ of degree $n$. Their poles in $D_{p}$, those zeroes of $q_{n}$ within $D_{p}$, accumulate (in some sense) to the singularities $z_{k p}$ of $f_{p}$ as $n$ increases, which is a deep result from potential theory established in [7]. Related resolution schemes are briefly described in [9] again. Hence, computing the zeroes of $q_{n}$ for suitable values of $n$ allows us to efficiently estimate the quantity $K$ of sources and to approximately localize the singularities $z_{k p}$. Indeed, one first increases the degree $n$ until the value of the approximation criterion (the quadratic error on $T_{p}$ ) is small enough on $T_{p}$ (or stationary): this furnishes an estimation of $K$, a nice feature of this scheme. Then, for such a degree $n$, one compute the solution $p_{n} / q_{n}$ and its $n$ poles, which are close to $z_{k p}$. Similarly, one computes best rational approximants with $m$ triple poles within $D_{p}$, represented as rationals $p_{3 m} /\left(q_{m}\right)^{3}$ in the above criterion. It appears that a single triple pole $(m=1)$ already approximates well enough the singularities $z_{k p}$. This property is established in [9, Prop. 1] for the case $K=1$ of a single source. It also numerically holds for $K=2$ or more, as illustrated by figure 6 , where the algorithm is run by the software FindSources3D [12]. We see there that in northern and southern planar sections $D_{p}$, the single triple pole is close to the one of the two singularities $z_{k, p}(k=1,2)$ which accounts for the closest source. This property furnishes an estimate of the quantity $K$ of sources from the behaviour of $m=1$ triple pole (in the present example, $K=2$ ), and - more approximately - there locations. A further rational approximation step must then be performed similarly, looking for triple poles at exact degree $m=K$ (see figure 7 ).

\section{From $2 D$ to $3 D$}

The above planar rational approximation algorithm is then run at degree $m=$ $K=2$ for planes $\Pi$ along 12 different directions. Figure 7 shows the 12 corresponding views from above of the singularities $\left(z_{k p}\right)$. These are estimated using the 2 triple poles computed in sections $\Pi_{p}$, for some values of $p$, together with a further step accounting for the last sum in expression (10). In some of the 12 pictures, the estimated singularities are located along 2 lines. We then select these most significant directions $\Pi$. The selected series of estimated $\left(z_{k p}\right)$ then approximately intersect within the ball $\Omega_{0}$ at the sources locations $C_{k}$, as they should exactly do, see figure 8 . We finally run a last clustering step, in order to refine the estimation. 


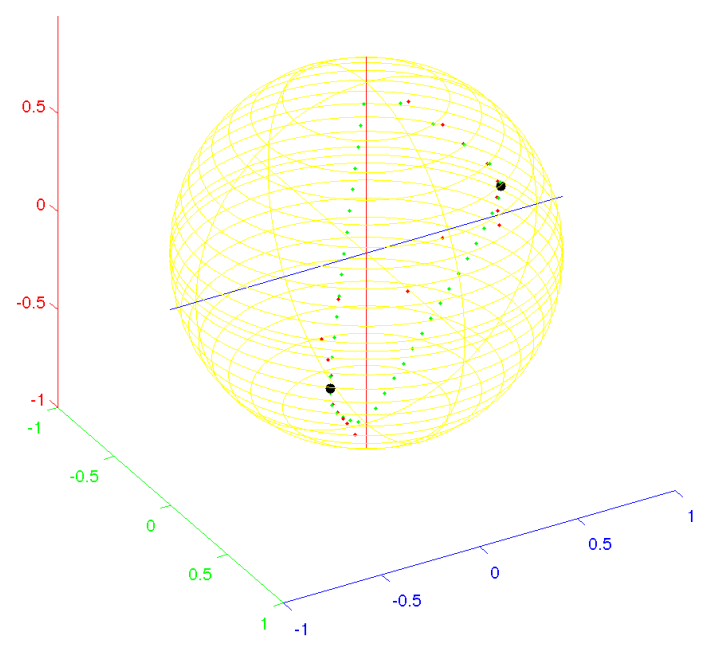

Figure 6: Sources $C_{k}$ in $\Omega_{0}$ (big black dots), singularities $\left(z_{k p}\right)$ (small green dots), for $k=1,2$, single triple pole (small red dots) in 21 parallel planar sections $\Pi_{p}$.

Source estimation algorithm, in short

- Input: spherical meshed geometry of $S_{0}$ and pointwise values of the singular part $\Psi_{S}$ of the potential (or coefficients of a spherical harmonic expansion; $\Psi_{S}$ can be computed from $\boldsymbol{\Psi}$ and $\frac{\partial \Psi}{\partial n}$ ); an integer $P$ (quantity of parallel slices $\Pi_{p}$ for each of the 12 planar sections $\Pi$ ).

- For each section $\Pi$, get $f_{p}$ on $T_{p}$, for $p=1, \cdots, P$.

- For some section $\Pi$, compute its best rational approximant with a single triple pole in $D_{p}, p=1, \cdots, P$, and estimate the quantity $K$ of sources.

- For each section $\Pi$ :

- Compute the best rational approximant to $f_{p}$ on $T_{p}$ with $m=K$ triple poles in $D_{p}$, for $p=1, \cdots, P$,

- estimate the singularities $\left(z_{k p}\right), k=1, \cdots K$.

- Cluster the computed singularities, and find estimates of $C_{k}, k=1, \cdots, K$.

- Output: estimated source locations $C_{k}, k=1, \cdots, K$.

Note that, once the source locations $C_{k}$ are estimated, their moments $p_{k}$ can be recovered by computation of the residues of the functions $f_{p}$ in the selected planar sections. 

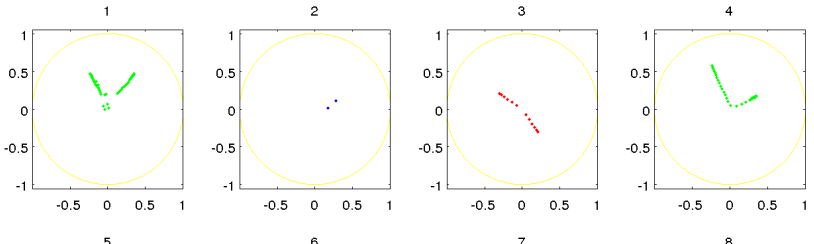

6
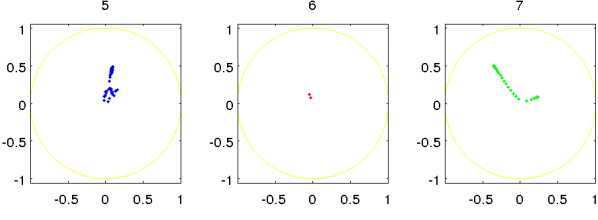

10
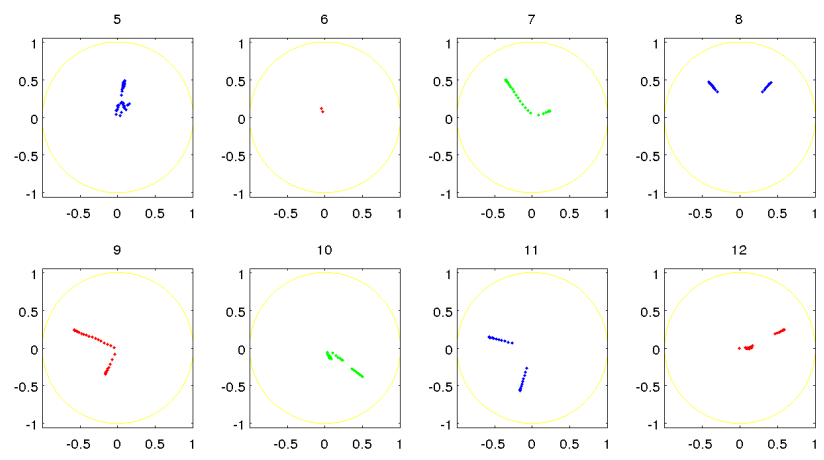

12

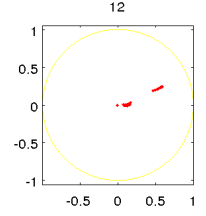

Figure 7: For 12 different planes $\Pi$, views from above of superimposed estimated singularities $\left(z_{k p}\right), k=1,2, p \in\{1, \cdots, 21\}$, using 2 triple poles.

\section{Numerical illustrations}

For figures $8,9,10$, the numerically generated data on $S_{0}$ correspond to $\mathbf{J}$ as in (9) and $K=2$ sources $C_{1}=(.2, .3, .4), C_{2}=(-.3,-.2, .4)$. Figures 9 and 10 show actual and estimated sources and moments. Though a small noise was added to the cortical data, the results however remain good enough, with a small localization error for the sources, as expected. In figure 10, the result is shown in a more realistic geometry (from MRI data, then translated on spheres).

These numericals illustrate the robustness and the efficiency of the involved identification schemes. They were all performed with the software FindSources3D [12]. It typically takes a few minutes to compute a solution to the full inverse EEG problem (on a linux laptop), from $L=128$ electrodes values to the estimation of $K=2$ sources, with $P=21$ planar sections in 12 different directions. 


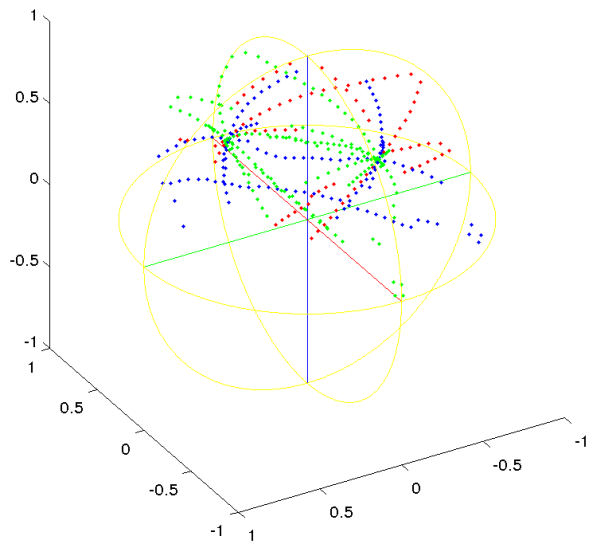

Figure 8: Superposition of estimated singularities in various slicing directions; the coloured series (lines) of singularities intersect at (next to) the sources.

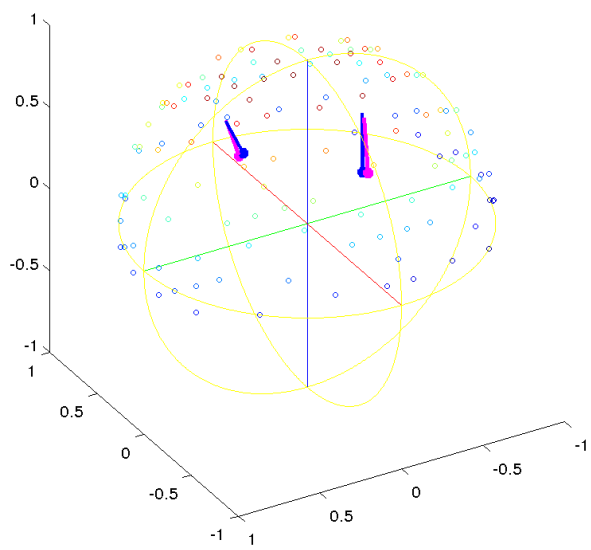

Figure 9: $K=2$ actual and estimated sources and moment, spherical geometry. 


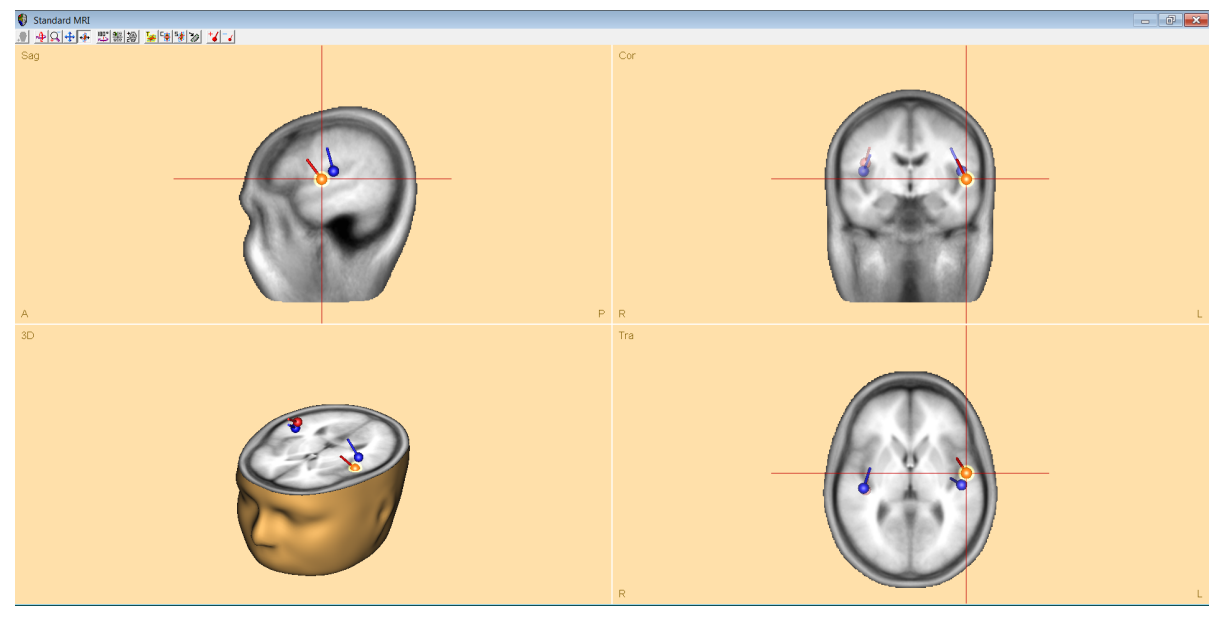

Figure 10: $K=2$ actual and estimated sources and moments, realistic geometry, with the courtesy of BESA GmbH.

\section{Conclusion}

Beyond the many inverse boundary value problems that arise in medical imaging, we discussed source recovery issues for EEG. In a spherical layered geometric head model, we presented a robust resolution scheme (observer) in order to estimate the source locations from the measured boundary data. It consists in a best rational approximation procedure on planar sections, coupled with a boundary element method (BEM) for preliminary data transmission steps. More realistic geometries can be considered with similar techniques, though the numerical complexity increases (several planar singularities may be associated to a single source) [17].

Further uniqueness / identifiability properties for geometric inverse problems of singularity localization (they may be sources, defaults, cracks) from boundary data are currently under study. In particular, a source term $\mathbf{J}$ is said to be silent in $\Omega_{0}$ if it not visible from outside $\Omega_{0}$, and produces a potential that vanishes there: $\boldsymbol{\Psi}=0$ on $\mathbb{R}^{3} \backslash \overline{\Omega_{0}}$. Silent (non-observable) sources for the homogeneous EEG inverse problem are analyzed (they are never pointwise). Taking the time variable into account within the model should also be done, using infinite-dimensional linear system theory [19].

A somehow similar model is also valid for the magnetic potential, and the inverse source problem in magnetoencephalography (MEG) can be tackled as well. There, the measured data are pointwise values of the radial component of the magnetic field, taken on part of a sphere located at some height above the scalp. Simultaneous EEG-MEG signals recordings should be available, which will improve the source estimation. Analogous inverse source problems also appear in geosciences and paleomagnetism [2], or for other physical potentials solutions to Newton's equations. 
Concerning electric impedance tomography (EIT) related issues, the conductivity $\sigma$ itself is unknown in (1) and is one of the quantities to be recovered (a question related to Calderón's inverse problem). One may then use the normal current $\mathbf{u}$ applied by electrodes on the scalp as an effective control, in order to estimate the conductivity values and to look for some optimal location of its support.

Acknowledgements: Many thanks to my main collaborators, L. Baratchart (INRIA Sophia Antipolis, Team APICS), M. Clerc (INRIA S.A., Team ATHENA), T. Jordanov (BESA GmbH), J.-P. Marmorat (Mines ParisTech, CMA, S. A.), T. Papadopoulo (INRIA S.A., Team ATHENA), and to the reviewers for their constructive suggestions.

\section{References}

[1] G. Alessandrini, L. Rondi, E. Rosset, S. Vessella, The stability for the Cauchy problem for elliptic equations, Inverse Problems, 25 (2009).

[2] E. Andrade-Lima, L. Baratchart, D. Hardin, E.B. Saff, B. Weiss, Characterizing kernels of operators related to thin plate magnetizations via generalizations of Hodge decompositions, Inverse Problems, 29 (2013).

[3] B. Atfeh, L. Baratchart, J. Leblond, J.R. Partington, Bounded extremal and Cauchy-Laplace problems on the sphere and shell, J. Fourier Analysis \& Applications, 16, 177-203 (2010).

[4] L. Baratchart, J. Leblond, J.-P. Marmorat, Inverse source problem in a 3D ball from best meromorphic approximation on 2D slices, Elec. Trans. Num. Anal., 25, 41-53 (2006).

[5] L. Baratchart, J. Leblond, J.R. Partington, N. Torkhani, Robust identification in the disc algebra from band-limited data, IEEE Trans. Automatic Control, 42 (1997).

[6] L. Baratchart, M. Olivi, F. Wielonsky, On a rational approximation problem in the real Hardy space $H_{2}$, Theoretical Computer Science, 94, 175-197 (1992).

[7] L. Baratchart, H. Stahl, M. Yattselev, Weighted extremal domains and rational approximation, Advances in Maths, 229, 357-407 (2012).

[8] G. Chen, J. Zhou, Boundary elements methods, Academic Press (1992).

[9] M. Clerc, J. Leblond, J.-P. Marmorat, T. Papadopoulo, Source localization in EEG using rational approximation on plane sections, Inverse Problems, 28, 055018 (2012).

[10] R. Dautray, J.-L. Lions, Analyse mathématique et calcul numérique pour les sciences er les techniques, vol. 2, Masson (1987). 
[11] A. El Badia, T. Ha Duong, An inverse source problem in potential analysis, Inverse Problems, 16, 651-663 (2000).

[12] M. Clerc, J. Leblond, J.-P. Marmorat, T. Papadopoulo, Software FindSources3D, http://www-sop.inria.fr/apics/FindSources3D/

[13] V. Isakov, Inverse problems for partial differential equations, Applied Mathematical Sciences, 127, Springer (1998).

[14] J.D. Jackson, Classical Electrodynamics, Wiley \& sons (1998).

[15] D. Kandaswamy, T. Blu, D. Van De Ville, Analytic sensing: Noniterative retrieval of point sources from boundary measurements, SIAM J. Scientific Computing, 31 (2009).

[16] J. Kybic, M. Clerc, T. Abboud, O. Faugeras, R. Keriven, T. Papadopoulo, A common formalism for the integral formulations of the forward EEG problem, IEEE Trans. Medical Imaging, 24, 12-28 (2005).

[17] J. Leblond, C. Paduret, S. Rigat, M. Zghal, Source localization in ellipsoids by best meromorphic approximation in planar sections, Inverse Problems, 24(3), 035017 (2008).

[18] M. Scherg, T. Bast, P. Berg, Multiple source analysis of interictal spikes: goals, requirements, clinical value, J. of Clinical Neurophysiology, 16, 214-238 (1999).

[19] M. Tucsnak, G. Weiss, Observation and Control for Operator Semigroups, Birkhäuser (2009).

[20] S. Vessella, Locations and strengths of point sources: stability estimates, Inverse Problems, 8 (1992).

[21] M. Zghal, Problèmes inverses pour l'équation de Laplace en dimension 3, application l'EEG, PhD thesis, ENIT, Univ. Tunis El Manar (2010). 